\title{
Fast hybrid particle-in-cell technique for pulsed-power accelerators
}

\author{
D. R. Welch, ${ }^{1}$ N. Bennett, ${ }^{2}$ T. C. Genoni, ${ }^{1}$ C. Thoma, ${ }^{1}$ and D. V. Rose ${ }^{1}$ \\ ${ }^{1}$ Voss Scientific, LLC, Albuquerque, New Mexico 87108, USA \\ ${ }^{2}$ Sandia National Laboratories, Albuquerque, New Mexico 87185, USA
}

(Received 24 July 2020; accepted 19 October 2020; published 3 November 2020)

\begin{abstract}
Hybrid-implicit particle-in-cell (PIC) algorithms permit the simulation of complex problems involving both kinetic and fluid plasma regimes over large spatial and temporal scales. Fluid electrons can be computationally fast where and when fluid assumptions are valid. Additional flexibility is obtained if discrete PIC macroparticles, with velocities advanced by either fluid or kinetic equations, are permitted to dynamically migrate between the two descriptions based on phase space criteria. Ideally, these migrations result in energetic particles treated kinetically and dense thermal plasma particles as a fluid. With an energy-conserving particle advance, resolution of the plasma Debye length is not required for numerical accuracy or stability. For pulsed-power applications, the simulation time step is usually constrained by the electron cyclotron frequency, not the more restrictive plasma frequency. A new implicit technique permits accurate particle orbits even at highly underresolved cyclotron frequencies. Thus, greater temporal and spatial scales can be accurately modeled relative to conventional PIC techniques. In this paper, we describe the hybrid PIC technique and fully electromagnetic, hybrid simulations of plasma evolution and current shunting in an idealized accelerator designed for driving a $Z$-pinch load. The dynamics of electrode heating, electron transport, and surface contaminant evolution are studied in a series of relativistic hybrid-implicit PIC simulations. These dynamics can lead to the shunting of current before reaching the $Z$-pinch load, thus degrading load performance. Examining two previously published power flow problems, we compare results from fully kinetic, multifluid, and hybrid kinetic-fluid simulations and discuss the computational performance of these three options. The key thrust of the work is to identify possible computational acceleration, through hybrid methods, required for accelerator understanding and design.
\end{abstract}

DOI: 10.1103/PhysRevAccelBeams.23.110401

\section{INTRODUCTION}

The particle-in-cell (PIC) method [1] is the workhorse of lower-density charged particle simulation for pulsed-power systems. The method solves the Boltzmann-Maxwell equations, where the particle velocity distribution function is represented by discrete velocities of macroparticles. For relativistic particle dynamics in multimegavolt accelerators, the standard momentum-conserving, explicit algorithm adequately describes particle transport, including electron sheath flows in magnetically insulated transmission lines (MITLs), when the plasma and cyclotron frequencies and the plasma Debye length are resolved (see, for example, Ref. [2] and references therein). As the energy

Published by the American Physical Society under the terms of the Creative Commons Attribution 4.0 International license. Further distribution of this work must maintain attribution to the author(s) and the published article's title, journal citation, and DOI. densities in pulsed-power systems have increased, so have the electrode surface temperatures. Ohmic heating is the primary reason, but particle impact and radiation deposition also contribute. This heating is important, because it introduces additional physics that must be modeled including rapid desorption of surface contaminants. After desorption, these contaminant materials are ionized through impact with energetic charged particles. The resulting electrode surface plasma is significantly denser than the electron flow and has been shown to impact accelerator performance $[3,4]$. Simulating these thermal plasmas is important but beyond the capability of traditional PIC techniques.

In this paper, we present a hybrid-implicit PIC technique to more rapidly simulate the relativistic and thermal particle populations present in high-power systems. A recent paper [5] detailing a new kinetic, implicit method has demonstrated the efficacy of the PIC method for modeling stressing high-density magnetized plasmas. The new magnetic implicit method is more stable and accurate when underresolving the cyclotron frequency. Here, the goals are to develop an accurate evaluation of the plasma physics and 
a fast method for evaluating new designs. First discussed in Ref. [6], the hybrid technique described here uses macroparticles advanced with both kinetic equations and fluid equations similar to the technique of Brackbill and Ruppel [7] and recently in Ref. [8]. The sum of these representations constitutes the complete six-dimensional distribution function. Fluid representations are better suited to describing the dense plasma regions near the electrode surfaces, while kinetic representations are necessary to describe details of the lower-density sheath flow. It can be advantageous to include both descriptions and/or allow macroparticles to change their governing equations dynamically such that the computationally faster but valid description is used. We refer to this technique in which the original equations governing kinetic and fluid particles are retained as "particle migration hybrid" (PMH).

We demonstrate the kinetic, fluid, and hybrid techniques in two previously published problems relevant to the highpower Sandia National Laboratories' 26-MA $Z$ accelerator [9-12]. In idealized three-dimensional and highly resolved two-dimensional simulations, the currents from three pulsed-power transmission lines, operated as MITLs, are added in a convolute to drive a $Z$-pinch load. Results from the fluid and hybrid techniques are compared to the fully kinetic treatment. While the electron sheath dynamics are faithfully captured only in the kinetic treatment, the current loss during transport is similar in each treatment, indicating the multifluid and PMH approaches have utility in these types of power flow simulations.

Details of the PIC hybrid formalism are described in Sec. II, and the accuracy of implicit solution is described in Sec. III. The surface physics used in the simulations is presented in Sec. IV. The simulation results of the idealized pulsed-power current adder region are then presented in Sec. V. The simulation methods of fully kinetic, multifluid, and hybrid are also applied to a highly magnetized "inner" MITL in Sec. VI. The benefits and drawbacks of each method are discussed. The summary and conclusions are given in Sec. VII.

\section{PIC FORMALISM FOR KINETIC AND FLUID PARTICLES}

The evolution of a plasma can be modeled with various degrees of approximation. For example, the Boltzmann equation

$$
\frac{\partial f}{\partial t}+v \cdot \nabla f+\frac{q}{m}(E+v \times B) \cdot \frac{\partial f}{\partial \gamma v}=\left(\frac{\partial f}{\partial t}\right)
$$

advances the six-dimensional (position $\mathbf{x}$, velocity $\mathbf{v}$, and relativistic factor $\gamma$ ) distribution function $f(\mathbf{x}, \mathbf{v})$ including the Lorentz force and a collision operator $\left(\frac{\partial f}{\partial t}\right)_{\text {coll }}$. In Gaussian units, the electromagnetic fields can be determined from Maxwell's equations:

$$
\begin{aligned}
\nabla \times \mathbf{B} & =\frac{4 \pi}{c} \mathbf{J}+\frac{1}{c} \frac{\partial \mathbf{E}}{\partial t}, \\
\nabla \times E & =-\frac{1}{c} \frac{\partial \mathbf{B}}{\partial t}, \\
\nabla \cdot E & =4 \pi \rho, \\
\nabla \cdot B & =0,
\end{aligned}
$$

where $c$ is the speed of light. Charge and current sources $\rho$ and $\mathbf{J}$, respectively, are determined from taking the zeroth and first velocity moments of $f$, respectively.

In the PIC technique, macroparticles carry attributes such as weight $w$, position $\boldsymbol{x}$, velocity $\boldsymbol{v}$, and internal energy $U$. In a given cell, $f$ is the sum of $N_{p}$ discrete kinetic particles and $L$ fluid particles, i.e., $f=f_{\text {kin }}+f_{\text {fld }}$, where in one spatial dimension

$$
f_{\text {kin }}=\sum_{n^{\prime}}^{N_{p}} w_{n^{\prime}} \delta\left(x-x_{n^{\prime}}\right) \delta\left(v-v_{n^{\prime}}\right)
$$

for kinetic particles and

$f_{\mathrm{fld}}=\sum_{l}^{L} w_{l} \delta\left(x-x_{l}\right)\left(\frac{m}{2 \pi k T}\right) \exp \left(-\frac{m(v-\bar{v})^{2}}{2 k T}\right)$

for fluid particles with average or fluid velocity $\bar{v}$ and $\delta$ is the Dirac delta function. The number density $N$ (number per length) in a computational cell from both kinetic and fluid particle weights $w$ is $N=\sum_{n^{\prime}}^{N_{p}} w_{n^{\prime}} \delta\left(x-x_{n^{\prime}}\right)+$ $\sum_{l}^{L} w_{l} \delta\left(x-x_{l}\right)$. Similarly, the temperature (and other moments) is calculated using a weighted average for kinetic particles as $T_{\text {kin }}=\frac{1}{3} \sum_{n^{\prime}}^{N_{p}} w_{n^{\prime}} m_{n^{\prime}} v_{n^{\prime}}{ }^{2} \delta\left(x-x_{n^{\prime}}\right) /$ $\sum_{n^{\prime}}^{N_{p}} w_{n^{\prime}} \delta\left(x-x_{n^{\prime}}\right)$ and for fluid particles as $T_{\text {fld }}=$ $\sum_{l}^{L} w_{l} T_{l} \delta\left(x-x_{l}\right) / \sum_{l}^{L} w_{l} \delta\left(x-x_{l}\right)$.

The charge density (and current) at grid index $i(i+1 / 2)$ is calculated from charge state $q$ and the moments of $f$ given by

$$
\rho^{i}=\sum_{n^{\prime}}^{N_{p}} q_{n^{\prime}} w_{n^{\prime}} S\left(x^{i}-x_{n^{\prime}}\right)+\sum_{l}^{L} q_{l} w_{l} S\left(x^{i}-x_{l}\right)
$$

and

$$
\begin{aligned}
J_{x}^{i+1 / 2}= & \sum_{n^{\prime}}^{N_{p}} q_{n^{\prime}} w_{n^{\prime}} v_{n^{\prime}} S\left(x^{i+1 / 2}-x_{n^{\prime}}\right) \\
& +\sum_{l}^{L} q_{l} w_{l} \bar{v}_{l} S\left(x^{i+1 / 2}-x_{l}\right) .
\end{aligned}
$$

The shape function $S(x)$ can be either the standard bilinear interpolation or a cloud-in-cell shape function. The mesh positions of the Yee fields [13] and distribution 
$(\rho, N, T, U, \Delta U, \Delta \psi \boldsymbol{v})^{i, j+1} \quad\left(E_{x}, J_{x}, P_{x}, v_{x}\right)^{i+1 / 2, j+1} \quad(\rho, N, T, U, \Delta U, \Delta \mathcal{w})^{i+1, j+1}$

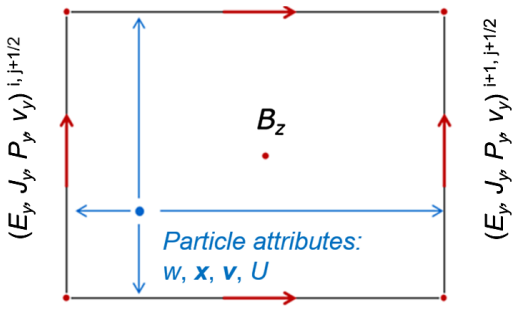

$(\rho, N, T, U, \Delta U, \Delta \gamma)^{i, j} \quad\left(E_{x}, J_{x}, P_{x}, v_{x}\right)^{i+1 / 2, j} \quad(\rho, N, T, U, \Delta U, \Delta \gamma)^{i+1, j}$

FIG. 1. The positions of the distribution moments $(\rho, N, v, P$, $U$, and $T$ ), electromagnetic fields, and currents $J$ are plotted on a representative $2 \mathrm{D}$ rectangular mesh in $(x, y)$ Cartesian geometry with $I$ and $j$ indices. Also plotted is a representative particle carrying attributes which are bilinearly interpolated to the full and edge position on the grid. Changes in internal energy and momenta are then interpolated back to particles.

moments are shown in Fig. 1 for a two-dimensional example. For the hybrid technique, a charge- and energy-conserving technique using a first-order shape function [14], similar to that discussed in Refs. [15,16]. This provides adequate smoothing of particle noise. The energy conservation for all simulations presented here is better than $5 \%$.

We construct augmented equations of motion (EOM) and energy equations to handle momentum and energy transfer between fluid and kinetic species (each designated by $\alpha$ or $\beta$ ). While collisions between kinetic species are handled with a binary scattering operator $\mathbf{F}_{\alpha, \beta}$ [17-19], we use fluidlike terms to handle momentum and energy transfer between a fluid species and other fluid or kinetic species [14]. The hybrid EOM advancing the macroparticles' momenta including the Lorentz force, pressure, and scattering terms is then

$$
\begin{aligned}
m_{\alpha} \frac{d \gamma \mathbf{v}_{\alpha}}{d t}= & +q_{\alpha}\left(\mathbf{E}+\frac{\mathbf{v}_{\alpha}}{c} \times \mathbf{B}\right) \quad(\alpha \text { is fluid or kinetic }) \\
& -\frac{\nabla P_{\alpha}}{n_{\alpha}} \quad(\alpha \text { is fluid only }) \\
& -\gamma m_{\alpha} \nu_{\alpha, \beta}\left(\overline{\mathbf{v}}_{\alpha}-\overline{\mathbf{v}}_{\beta}\right) \quad(\alpha \neq \beta, \alpha \text { or } \beta \text { is fluid }) \\
& +\mathbf{F}_{\alpha, \beta} \quad(\alpha \text { and } \beta \text { are kinetic }),
\end{aligned}
$$

where the specie's pressure is $P_{\alpha}$, the cell-averaged velocity is $\overline{\mathbf{v}}$, and $\nu_{\alpha, \beta}$ is the Spitzer collision frequency. The binary scattering operator advances momentum and energy between and within kinetic species only. Similarly, the terms on the rhs of Eq. (7) are not applied when no affected species are present.
In the PIC fluid method, convection of moments (mass, charge, $\boldsymbol{v}$, and $U$ ) is achieved through the particle position update, while other transport, such as thermal diffusion and interspecies collisions, is accomplished on the grid (see Fig. 1). If fluid particles are present, the hybrid method requires an additional energy equation for each species. An equation considering rhs terms for $P d V$ work, thermal conduction, thermal equilibration, and Ohmic heating is

$$
\begin{aligned}
N_{\alpha} \frac{d U_{\alpha}}{d t}= & -P_{\alpha} \nabla \cdot \mathbf{v}_{\alpha}+\nabla \cdot \kappa_{\alpha} \nabla T_{\alpha} \quad(\alpha \text { isafluidspeciesonly }) \\
& +N_{\alpha} \sum_{\beta} \frac{3 m_{\alpha} \nu_{\beta \alpha}}{m_{\alpha}+m_{\beta}}\left(T_{\beta}-T_{\alpha}\right) \\
& +N_{\alpha} \sum_{\beta} \nu_{\beta \alpha} \frac{m_{\alpha} m_{\beta}}{m_{\alpha}+m_{\beta}}\left(\bar{v}_{\beta}-\bar{v}_{\alpha}\right)^{2} \\
& (\alpha \neq \beta, \alpha \text { or } \beta \text { is a fluid species })
\end{aligned}
$$

where $U_{\alpha}$ and $T_{\alpha}$ are the species internal energy and temperature, respectively, and $\kappa_{\alpha}$ is the thermal conductivity. Note the Ohmic heating term gives heating only when two species have different velocities, as is the typical case of an induced electron current scattering with stationary plasma ions. The relationship between the internal energy and temperature is

$$
\frac{\partial U_{\alpha}}{\partial T_{\alpha}}=C_{v \alpha},
$$

where $C_{v \alpha}$ is the specific heat at constant volume. Although an equation of state can be used for fluids, an ideal gas is usually assumed for kinetic species. As with Eq. (7), the terms on the rhs are not applied when no affected species are present. If $\alpha$ and $\beta$ are both kinetic, the binary scatting operator is invoked for both momentum and energy transfer.

With the hybrid method, Eqs. (7)-(9) are solved by first convecting relevant moments by advancing particle positions and momenta with the Lorentz force [the first term on the rhs of Eq. (7)]. Next, the particle moments are scattered out to the grid, where detailed transport of energy and momentum is completed with these changes gathered back to the particles. Equation (8) is solved after the particles are pushed. The appropriate particle distribution moments, such as $N$ and $U$ as shown in Fig. 1, are then scattered (bilinearly interpolated) to the grid-some to grid nodes $(i)$ and some to grid edges $(i+1 / 2)$. In a one-dimensional example (advancing a time step $\Delta t$ in $x$ with grid index $i$ ), we difference Eq. (8) as follows: 


$$
\begin{aligned}
\frac{\Delta U_{\alpha}^{n+1 / 2, i}}{\Delta t}= & -\frac{P_{\alpha}}{N_{\alpha}} \frac{v_{x \alpha}^{n+1 / 2, i+1 / 2}-v_{x \alpha}^{n+1 / 2, i-1 / 2}}{\Delta x^{i}}+\frac{1}{N_{\alpha}} \sum_{\beta} \frac{3 m_{\alpha} \nu_{\beta \alpha}}{m_{\alpha}+m_{\beta}}\left(T_{\beta}^{n+1 / 2, i}-T_{\alpha}^{n+1 / 2, i}\right) \\
& +\frac{\frac{\kappa_{\alpha}}{\Delta x^{i+1 / 2}}\left(T_{\alpha}^{n+1 / 2, i+1}-T_{\alpha}^{n+1 / 2, i}\right)-\frac{\kappa_{\alpha}}{\Delta \Delta^{i-1 / 2}}\left(T_{\alpha}^{n+1 / 2, i}-T_{\alpha}^{n+1 / 2, i-1}\right)}{\Delta x^{i}}+\sum_{\beta} \nu_{\beta \alpha} \frac{m_{\alpha} m_{\beta}}{m_{\alpha}+m_{\beta}}\left(\bar{v}_{x \beta}^{n+1 / 2, i}-\bar{v}_{x \alpha}^{n+1 / 2, i}\right)^{2},
\end{aligned}
$$

yielding the change in the internal energy $\Delta U_{\alpha}^{n+1 / 2, i}$. Transport quantities include the thermal conductivity $\kappa_{\alpha}$ and collision frequencies $\nu_{\beta \alpha}$ which must be symmetric such that energy exchange between species is conserved as described in Ref. [20]. In the older direct implicit method, the velocities are advanced to the $n+1 / 2$ step and could be used directly [14]. To be consistent with the new magnetic implicit finite differencing (discussed in detail in Ref. [5] with more details concerning orbit accuracy in Sec. III) where particle positions and momenta are both advanced to the full time step level $n+1$, we must average the velocities back to the $n+1 / 2$ time level for proper centering, i.e., $v^{n+1 / 2}=0.5\left(v^{n}+v^{n+1}\right)$. This centering is important, because the $P d V$ and Ohmic terms using velocities are usually dominant. The change in temperature determined from Eqs. (9) and (10) is gathered back to the macroparticles via bilinear interpolation where the particle $U_{\alpha}$ is advanced.

Similarly, the pressure acceleration and exchange of momenta between species are calculated on grid as illustrated in Fig. 1. Having previously applied the Lorentz term in Eq. (7), we difference the remaining terms in Eq. (7) as

$$
\begin{aligned}
\Delta \gamma v_{x \alpha}^{n+1 / 2, i} \frac{m_{\alpha}}{\Delta t}= & -\frac{\nabla\left(P_{\alpha}^{n+1 / 2, i+1 / 2}-P_{\alpha}^{n+1 / 2, i-1 / 2}\right)}{N_{\alpha} \Delta x^{i}} \\
& -\gamma_{\alpha}^{n+1 / 2, i} m_{\alpha} \nu_{\alpha, \beta}\left(\overline{\mathbf{v}}_{\alpha}^{n+1 / 2, i}-\overline{\mathbf{v}}_{\beta}^{n+1 / 2, i}\right) \\
& +\mathbf{F}_{\alpha, \beta}^{n+1 / 2, i},
\end{aligned}
$$

yielding the change in momentum $\Delta \gamma v_{x \alpha}^{n+1 / 2, i}$ which is gathered back to the particles completing the momentum advance to the full step $n+1$. The individual particle momenta and internal energy are then advanced as discussed in Ref. [21] for fluid particles:

$$
\begin{aligned}
(\gamma u)^{n+1} & =(\gamma \bar{u})^{n+1 / 2}+\Delta \gamma v^{n+1 / 2}, \\
U^{n+1} & =U^{n+1 / 2}+\Delta U^{n+1 / 2},
\end{aligned}
$$

and kinetic particles:

$$
\begin{aligned}
(\gamma u)^{n+1}= & (\gamma \bar{u})^{n+1 / 2}+\Delta \gamma v^{n+1 / 2} \\
& +\left((\gamma u)^{n+1 / 2}-(\gamma \bar{u})^{n+1 / 2}\right) \sqrt{1+\frac{\Delta T^{n+1 / 2}}{T^{n+1 / 2}}} .
\end{aligned}
$$

As with kinetic macroparticles, the fluid macroparticles are created from surface interactions, such as space-charge-limited emission and desorption, and are destroyed or reflected when entering a surface. Furthermore, a Lagrangian push or Eulerian remap technique can be employed that reduces the fluid particle count to a single particle per cell [20]. This technique, while more dispersive, can be much computationally much faster and is used in simulations presented in this paper.

Because of the computational speed, it can be advantageous to use the multifluid descriptions if two key fluid assumptions are valid: Fluid particles move with their ensemble velocity, allowing no interpenetration of particles within the fluid, and the plasma has a Maxwellian distribution about the ensemble velocity. Given these assumptions, it is not recommended using fluids to model colliding plasmas as a single fluid if the collisional mean free path is long compared with spatial resolution [22]. Also, one would not use fluids to model beam-target fusion (fusion from a high-energy tail on the ion distribution dominates thermonuclear) [23]. In the PMH technique when the fluid assumption is breaking down or becoming valid, macroparticles of one EOM can migrate to the other EOM, exactly conserving moments. An example of a simple transition criterion is a particle kinetic energy (KE) threshold below which particles migrate to fluid and above which to kinetic macroparticles [24]. The energy threshold is a natural criterion for diodes and MITLs, since evolving high-density electrode plasmas are approximately Maxwellian and higher-energy charged particle electron and ion sheaths are typically not Maxwellian. Other criteria include thermal temperature, particle perveance [ratio of particle space charge to kinetic energy, $q w / m(\gamma-1)]$, and the ratio of thermal to kinetic or hydrodynamic energy $[\mathrm{kT} / \mathrm{m}(\gamma-1)]$. Fluid macroparticles migrating to kinetic are best treated by creating multiple kinetic macroparticles with energy sampling the thermal distribution and normalized such that $\sum_{n^{\prime}} \frac{1}{2 N_{p}} m\left(v_{n^{\prime}}-v_{l}\right)^{2}=\frac{3}{2} k T_{l}$, where $N_{p}$ kinetic particles are created for each fluid migrated. Kinetic macroparticles migrate all their momentum into the fluid. If the fluid macroparticle velocity deviates from the cell ensemble velocity (average fluid particle velocity in a cell), the energy difference increases the local fluid temperature. Thus, all quantities are conserved. 


\section{ORBIT ACCURACY OF MAGNETIC IMPLICIT SOLUTION}

The implicit scheme used here to advance both fluid and kinetic particles with the same time integration scheme is similar to the mature energy-conserving direct implicit PIC algorithm [14,21,25]. As discussed in Sec. II, we have adapted the more advanced magnetic implicit (MI) kinetic technique $[5,26]$ for use with multifluids. With this technique, the usual PIC limitations on the time step, namely, the need to resolve the cyclotron $\left(\omega_{c}\right)$ and plasma $\left(\omega_{p}\right)$ frequencies, are greatly relaxed. It is important to note that, for both kinetic and fluid descriptions in regimes where $\omega_{p} \Delta t>1$, high-frequency interactions are not temporally resolved. For example, one would not employ such a large time step if simulating the electron two-stream instability.

The MI algorithm is useful for very dense plasmas where high-frequency electron plasma oscillations can be ignored. Unlike most implicit (and explicit) algorithms, MI does not require resolution of the Debye length to avoid grid heating even when the time steps are small, $\omega_{p} \Delta t<1$. Additionally, the MI algorithm accurately represents spatial orbits in highly magnetized plasmas. Here, we justify the use of the magnetic implicit algorithm at the large time steps $\left(\omega_{c} \Delta t<9\right)$ employed in simulations discussed in this paper. We consider charged particle motion in a magnetic field in the $+z$ direction. We first illustrate purely circular motion in a magnetic field with no electric field and show that the MI algorithm preserves the particle momentum and Larmor radius exactly, regardless of the time step. A numerical example of the Larmor motion with $\omega_{c} \Delta t=$ $10 \pi$ follows. Next, we take up $E \times B$ drift motion relevant to MITL charged particle flow. A sample calculation gives the correct drift velocity and transverse extent of the quasicycloidal motion.

\section{A. Larmor motion}

Consider the geometry shown in Fig. 2 for Larmor circular motion of a particle in a magnetic field. From Ref. [26], the following equations advance the particle momentum $(p=\gamma \beta)$ and position:

$$
\begin{aligned}
& p^{n+1}=M_{1} p^{n}, \\
& x^{n+1}=x^{n}+c \Delta t \frac{p^{n}+p^{n+1}}{\gamma^{n}+\gamma^{n+1}},
\end{aligned}
$$

where $M_{1}$ is the rotation matrix,

$$
M_{1}=\frac{1}{1+\theta_{l}^{2}}\left(\begin{array}{cc}
1-\theta_{l}^{2} & 2 \theta_{l} \\
-2 \theta_{l} & 1-\theta_{l}^{2}
\end{array}\right)
$$

where $\theta_{l}=B \Delta t / 2 \gamma^{n}$. We have absorbed $q / m c$ into $B$ so that it has unit of $\mathrm{s}^{-1}$. Equation (14) yields

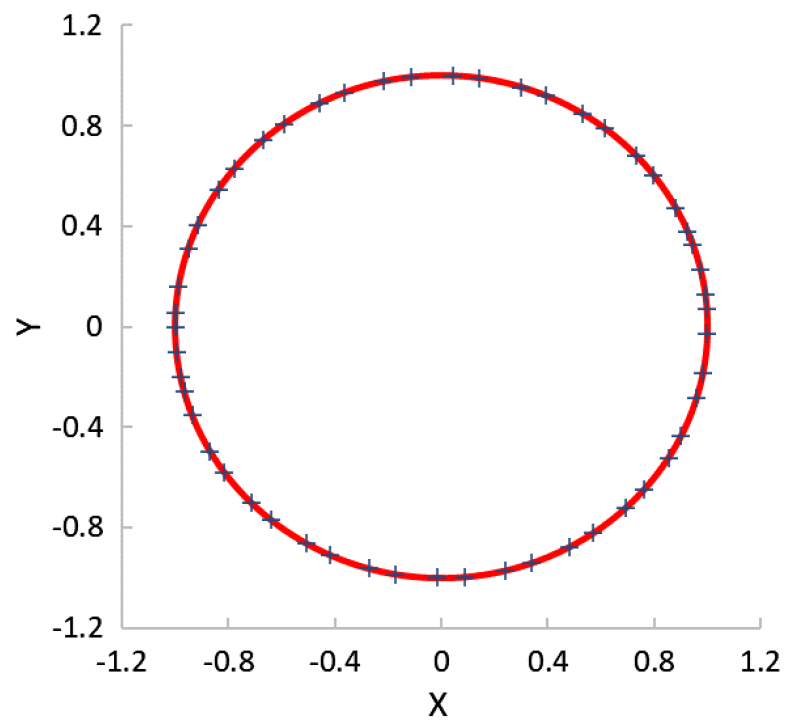

FIG. 2. For a charged particle with $r_{L}=1$ and $v_{x}$ $(o)=r_{L} \omega_{c}$, the charge particle motion in the $x-y$ plane is shown for the exact solution (red line) and MI calculation (+) with $\omega_{c} \Delta t=10 \pi$.

$$
x^{n+1}=x^{n}+\frac{c \Delta t\left(I+M_{1}\right) p^{n}}{\gamma^{n}+\gamma^{n+1}},
$$

where $I$ is the identity matrix. Since $M_{1}$ is an orthogonal matrix, it follows trivially that the magnitude of $p$ is preserved; i.e., $\left|p^{n+1}\right|=\left|p^{n}\right|$. It also follows that $\gamma^{n+1}=$ $\gamma^{n}$ and, thus, energy is conserved.

The Larmor radius of the circular orbit is

$$
r_{L}=\frac{p^{n} c \Delta t}{2 \gamma^{n} \theta_{l}}
$$

We note also that the center of the Larmor orbit is

$$
r_{c}=r^{n}+A \hat{p}^{n} r_{L},
$$

where $A$ is the antisymmetric matrix

$$
A=\left(\begin{array}{cc}
0 & 1 \\
-1 & 0
\end{array}\right)
$$

and $\hat{p}^{n}=p^{n} /\left|p^{n}\right|$ is the unit vector in the $p^{n}$ direction. Using Eqs. (13) and (14), Eq. (12) can be written as

$$
x^{n+1}=x^{n}+\theta_{l}\left(I+M_{1}\right) \hat{p}^{n} r_{L} .
$$

From Eqs. (14) and (15), we obtain

$$
r^{n}-r_{c}=M_{2} \hat{p}^{n} r_{L}
$$

where 


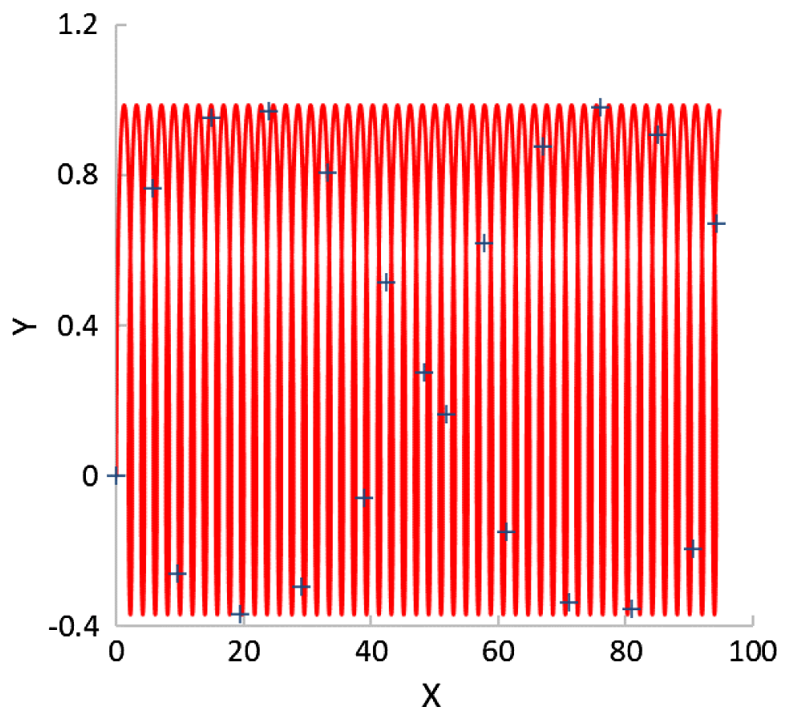

FIG. 3. For a charged particle with $r_{L}=1$ and $v_{x}(o)=0.2 c$, $v_{E x B}=0.1 c$, the charge particle motion in the $X-Y$ plane is shown for the exact solution (red line) and MI calculation (+) with $\omega_{c} \Delta t=5 \pi$.

$$
M_{2}=\frac{1}{1+\theta_{l}^{2}}\left(\begin{array}{cc}
2 \theta_{l} & \theta_{l}^{2}-1 \\
1-\theta_{l}^{2} & 2 \theta_{l}
\end{array}\right)
$$

Since $M_{2}$ is also an orthogonal matrix and $\hat{p}^{n}$ is a unit vector, we see immediately that $\left|r^{n}-r_{c}\right|=r_{L}$ and the exact Larmor radius is preserved. Given a $B_{z}$ and initial velocity $v_{x}=r_{L} \omega_{c}$ such that $r_{L}=1$, Fig. 2 compares the exact Larmor orbit in the $x-y$ plane and an MI calculation at $\omega_{c} \Delta t=10 \pi$. We see that $r_{L}$ is indeed conserved in the simulation, although the phase of the oscillation cannot be preserved because of the large time step.

\section{B. $E \times B$ drift motion}

To the previous example, we add an electric field in the $+y$ direction. This results in an $E \times B$ drift in the $+x$ direction, $v_{E \times B}=0.1 c$, as shown in Fig. 3. The MI calculation with $\omega_{c} \Delta t=5 \pi$ reproduces the correct drift velocity and transverse envelope of the $y$ motion. The large time step precludes replication of the correct phase. Thus, the MI method is ideal for high-current $Z$ accelerator simulations given its accurate representation of the Larmor radius and drift orbits at large time steps.

\section{SURFACE PHYSICS MODELING IN MITLS}

In a transmission line, there are initially no free current carriers and the pulsed electromagnetic wave increases the applied AK (anode-cathode) voltage and electric field stresses. For typical bare metal surfaces, electrons are emitted from the cathode above an electric field threshold of roughly $250 \mathrm{kV} / \mathrm{cm}$. As the threshold is exceeded, the surface breaks down, explosively yielding a surface plasma. In the electrode model used in the Chicago code (also available in the Lsp code) [6,14,27], the explosive breakdown of the cathode increases the surface temperature by $100^{\circ}$ (an adjustable parameter in the model). This increments the surface temperature, which is already increasing due to Ohmic heating and charged particle impact heating. The Ohmic heating model is described in Ref. [28], where the thermal conductivity and specific heat are assumed to be constant. After a surface temperature rise of roughly $400{ }^{\circ} \mathrm{C}$, the electrode plasma is desorbed thermally (using the Arrhenius equation discussed in Ref. [5]), ionizes and then provides a source of electrons or ions for acceleration across the AK gap. The plasma is assumed to be comprised of water molecules, which are a significant constituent of surface contaminants. A completely fragmented and ionized water plasma $\left(3 e^{-}, 2 \mathrm{H}^{+}, \mathrm{O}^{+}\right)$is produced within a cell of the material surface. The assumption of the model is that the energy required to ionize and dissociate the water is small (roughly 25-30 eV) compared with the gap voltage (of the order of $1 \mathrm{MV}$ ). Detailed smaller-scale breakdown simulations have begun with preliminary results that show this assumption leads to a more rapid ionization of the desorbed water (because the avalanche time is underestimated) but yields a qualitatively similar plasma profile delayed by $\sim 3$ ns. This desorption model uses the Temkin isotherm, which is based largely on the experimental results of $\mathrm{Li}$ and Dylla [29] and Dylla, Manos, and LaMarche [30], and the theoretical analysis of La Fontaine [31]. Discussed in detail in Ref. [5], we assume a $10^{-5}$ torr pressure giving one adsorbed monolayer of water (defined here as $8 \times 10^{15}$ molecules $/ \mathrm{cm}^{2}$ ) with an initial $0.83 \mathrm{eV}$ binding energy on stainless steel.

\section{APPLICATION OF HYBRID PIC TO A HIGH-POWER CURRENT ADDER}

An idealized representation of a high-power accelerator, modeled on Sandia National Laboratories' $Z$ machine, is used here to illustrate the application of the techniques described in Secs. II-IV. In the $Z$ accelerator, 36 pulsedpower modules are connected in parallel to four radial MITLs. The currents from these four MITLs are combined via an adder region, referred to as a post-hole convolute, into a single high-power inner MITL which drives the load. The electrodes of the convolute and inner MITL are particularly susceptible to the degrading effects of Ohmic and charged particle heating and subsequent plasma ablation, which can limit current delivery to the load. The idealized accelerator has all the physics considerations of the $Z$ machine, including low-density electron flow from explosive emission on the cathode and contaminant desorption from the electrode surfaces. This idealized geometry has been used previously to study the scaling of current loss under the assumption of quasiequilibrium operation using 3D PIC simulations of the radial MITLs and post-hole 


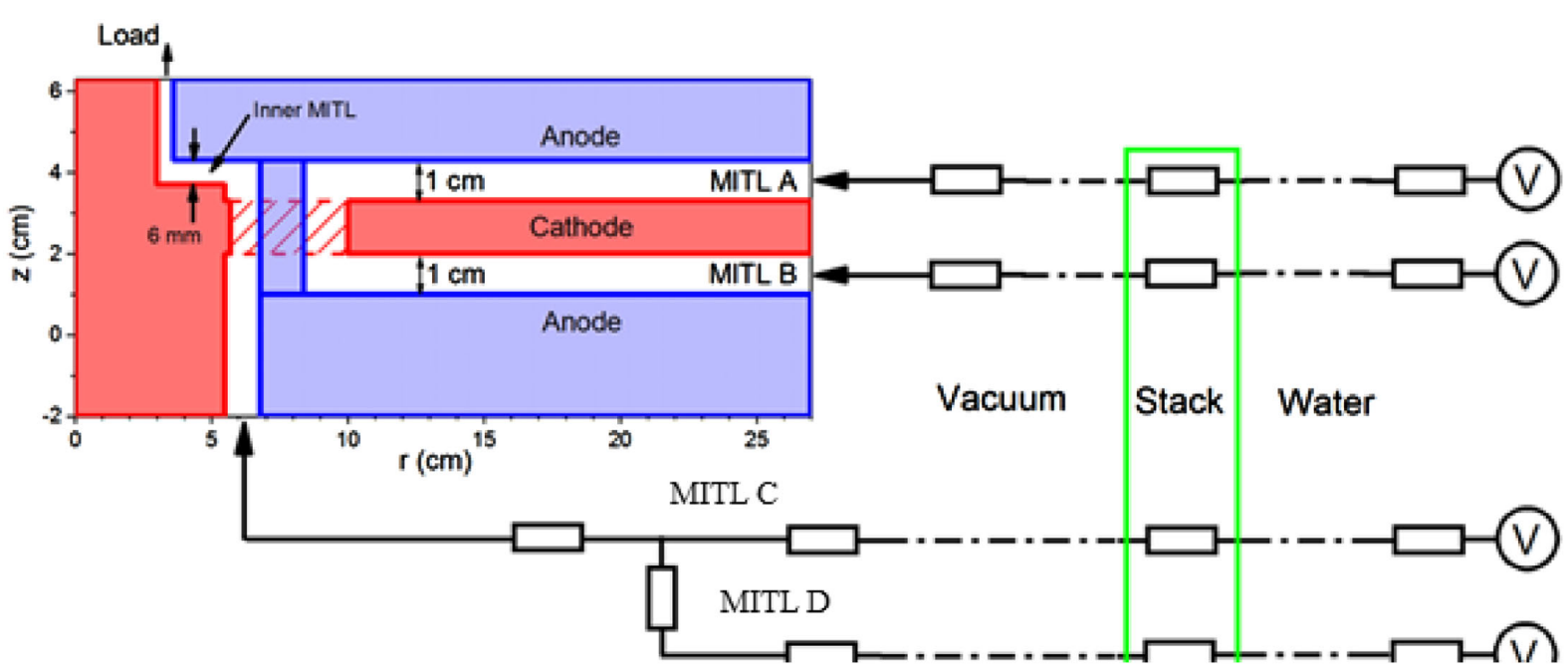

FIG. 4. A schematic of the 3D simulation is shown with the $Z$ circuit input and a short-circuit load.

convolute region. The losses were found to scale strongly with load impedance and the system vacuum pressure and were calculated to be as high as 1-2 MA.

The idealized geometry consists of three outer MITLs that add currents in a post-hole convolute, which then feeds the total current into a single inner MITL, as described in Ref. [32]. The geometry, illustrated in Fig. 4, is modeled in 3D cylindrical coordinates using Chicago. The voltage pulses, injected at three locations corresponding to the upstream boundaries of the outer MITLs, are modeled from a network-circuit representation of the $Z$ machine. Downstream from the convolute, a circuit takes the power from the inner MITL to the load (a short circuit is used here). While a full 3D representation would contain 12 anode posts, the simulation includes a pie wedge from a post center to midway between two posts, with symmetry planes at $\theta=0$ and $0.2618 \mathrm{rad}$. The spatial resolution is $100 \mu \mathrm{m}$ in the $r$ and $z$ directions, but $\theta$ is resolved more coarsely with only 20 cells. The time step is initially $0.67 \mathrm{ps}$ but adjusts dynamically such that $\omega_{c} \Delta t<9$ as the current rises for all simulations presented. Because of this frequency constraint, the collision rates are all resolved even in the dense electrode plasmas. We have confirmed convergence with a smaller time step $\left(\omega_{c} \Delta t<3\right)$ in kinetic simulations as well. We use the implicit and hybrid PIC techniques implemented in Chicago as described in Secs. II and III.

We ran four simulations of the geometry in Fig. 4 with the $Z$ voltage pulses using fully kinetic, multifluid, and all PMH hybrid (fluid and kinetic treatments for electrons and ions) and fluid electrons with PMH ions. The simulations include water desorption with electron, hydrogen ions, and oxygen ions (assumed in +1 state only). To gain any speed up over fully kinetic simulation, the hybrid method must keep the number of kinetic macroparticles to a minimum. Thus, the transition criterion for migration from fluid to kinetic for all three charged particles is $\mathrm{KE}>50 \mathrm{keV}$.
Lower KE values were tested with similar transport but yielded too many particles. Once particles transition to kinetic, few will thermalize before being lost to the walls.

The simulation electron densities are shown in Fig. 5 after $90 \mathrm{~ns}$ for the four simulation techniques. The kinetic simulation electrons exhibit characteristic vortices seen in outer radial MITLs and convolutes where the impedance is changing significantly as the electrons move downstream [33]. The multifluid and hybrid simulations have a similar overall sheath thickness with little evidence of vortices late in time as seen in the kinetic simulations. The key assumption in fluid description is that plasma motion is at the local mean or fluid velocity resulting in no interpenetration. The large magnetic fields in the $>$ MA currents later in time at smaller radii result in the order of $1 \mu \mathrm{m}$ electron cyclotron radius. Because of simulation spatial resolution of the order of $100 \mu \mathrm{m}$ and fluid assumptions, the relative velocities of the vortices can be underresolved and result in fluid heating. While at early times and larger radii, the fluid simulations do exhibit larger scale vortices, only kinetic particles can maintain the vortices near peak current. Downstream of the post $(r<6 \mathrm{~cm})$, the kinetic, multifluid, and hybrid simulation densities are all quite smooth at this time. As discussed in Refs. [5,34], the inner MITL region near the load exhibits complex kinetic instabilities and turbulent transport that might require kinetic representations for the plasma. This behavior is addressed in the next section.

All simulations show some small loss (tens of $\mathrm{kA}$ ) to the upstream post $(z \sim 3 \mathrm{~cm})$ described in Refs. [12,32], where the magnetic null is found (a channel of very small magnetic field due to the symmetry breaking of the post current). The current transported to the load at $z=6 \mathrm{~cm}$ and the difference between the current at the entrance to the MITL and the load current or loss current are plotted in Fig. 6. Up to roughly $65 \mathrm{~ns}$, the four simulations calculate the same load and loss currents to within $2 \%$ and 5\%, 


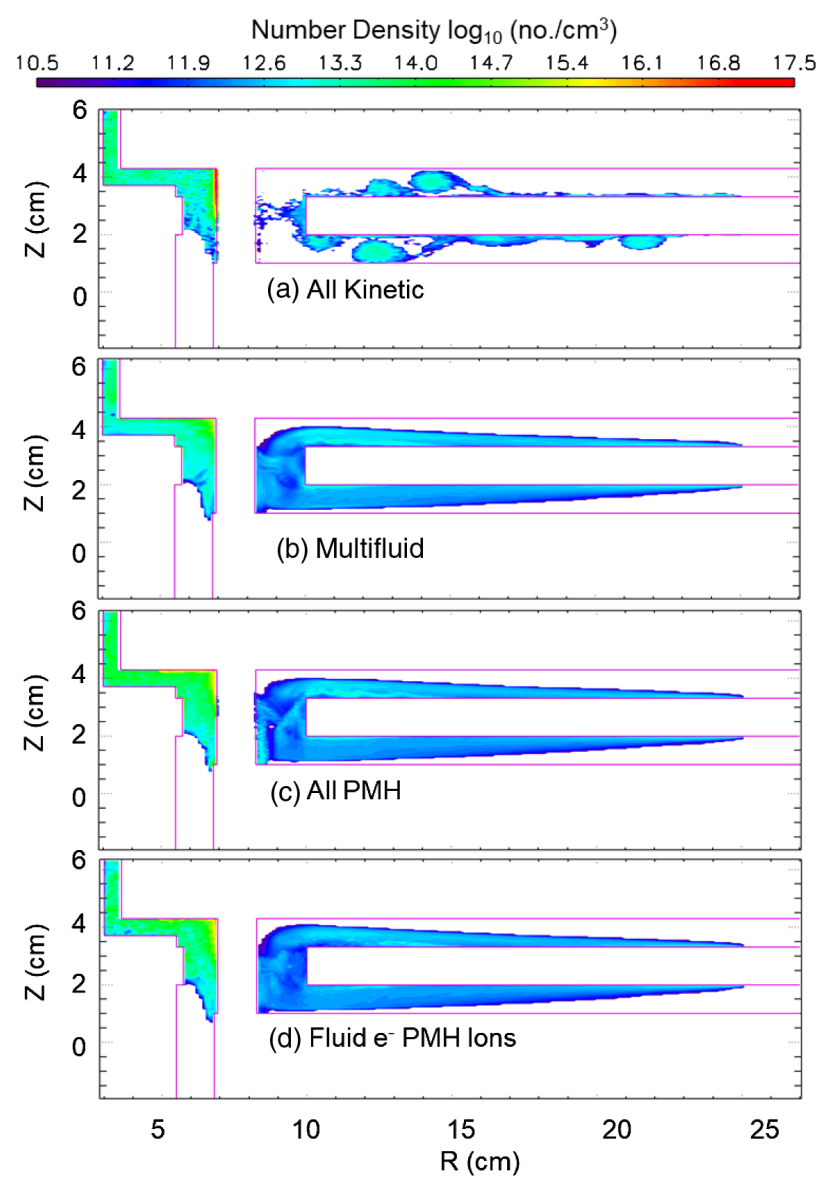

FIG. 5. The electron density is plotted $90 \mathrm{~ns}$ into the four simulations in the $\theta=0$ plane (center of post). The plot shows results from (a) all kinetic particles, (b) the multifluid or all fluid particles, (c) all PMH with fluid particles transitioning to kinetic above $50 \mathrm{keV}$, and (d) fluid electrons with PMH ions. All these simulations have dynamic time steps such that $\omega_{c} \Delta t<9$.

(a)

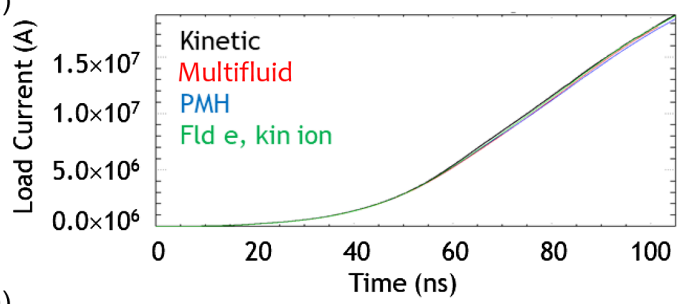

(b)

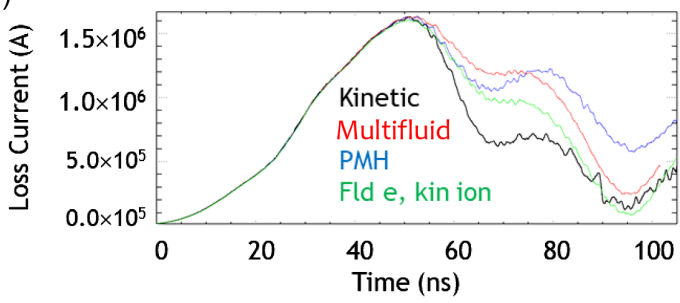

FIG. 6. The (a) load current calculated at $z=6 \mathrm{~cm}$ and (b) loss current (difference between total current entering the MITLs and the load current) from four simulations (kinetic, multifluid, $\mathrm{PMH}$, and fluid $e$ with $\mathrm{PMH}$ ions) using the geometry in Fig. 4 are shown. respectively. All calculate the same time of initial current loss peak at $53 \mathrm{~ns}$, where the voltage reaches $1.5 \mathrm{MV}$ and electron flow current is highest. This initial loss is mainly due to launched electron flow in the outer MITLs that is mostly lost in the convolute. The numerical agreement largely demonstrates that all numerical techniques are sufficiently accurate for modeling the flow current.

Because the downstream load is a short, the voltages plateau and the current continues to rise after $53 \mathrm{~ns}$, causing the electron flow current to fall. All simulations exhibit a smaller second peak in current loss due mainly to dense plasma motion just downstream of the post for $r<7 \mathrm{~cm}$, although the multifluid and PMH simulations' peak is higher because of small differences in the motion of the thermal plasma from the electrode. The reason for the difference is likely the larger dispersion seen in the Eulerian fluid description.

Note the better agreement in current loss between the all kinetic and fluid electron or PMH ion results shown in Fig. 6. This behavior suggests that the kinetic ion description is preferable to fluid later in time when the losses are small. While the observed differences in calculated densities between the techniques are interesting, the overall performance of each technique in simulating current transport to drive the load is similar, indicating the fluid and $\mathrm{PMH}$ approaches have utility in these types of power flow simulations.

The potential for computational speed up with the fluid and hybrid approaches make them desirable for designlevel calculations with speed up factors as high as 6 (relative to the all kinetic depending on the total particle number) observed so far. If you are stingy with the kinetic particle number as we were in the simulations presented, the multifluid speed up was a modest $20 \%$. These simulations required roughly 20000 processor hours on a Linux cluster using 20 conventional compute nodes. Each compute node has two 14-core Intel Xeon $2.6 \mathrm{GHz}$ processors and $128 \mathrm{~GB}$ of memory with a low-latency, FDR Infiniband interconnect (Fourteen Data Rate, $14 \mathrm{~Gb} / \mathrm{s}$ data rate per lane).

\section{APPLICATION OF HYBRID PIC TO A 26-MA MITL}

A series of power flow experiments on the $Z$ accelerator were performed to study losses in the system. Recent simulations of the inner MITL in these experiments have shown that the effects of strong magnetization can rapidly drive plasma into the gap, which leads to a Hall-like current loss mechanism [32]. We repeated the 2D simulations of these experiments using the four techniques used to simulation the convolute: kinetic, multifluid, $\mathrm{PMH}$, and fluid electrons with ion PMH. The kinetic result was described in detail in Ref. [5]. The inner MITL, modeled as a circuit in the previous section, connects the convolute and load as shown in the upper left in Fig. 4. To model the 


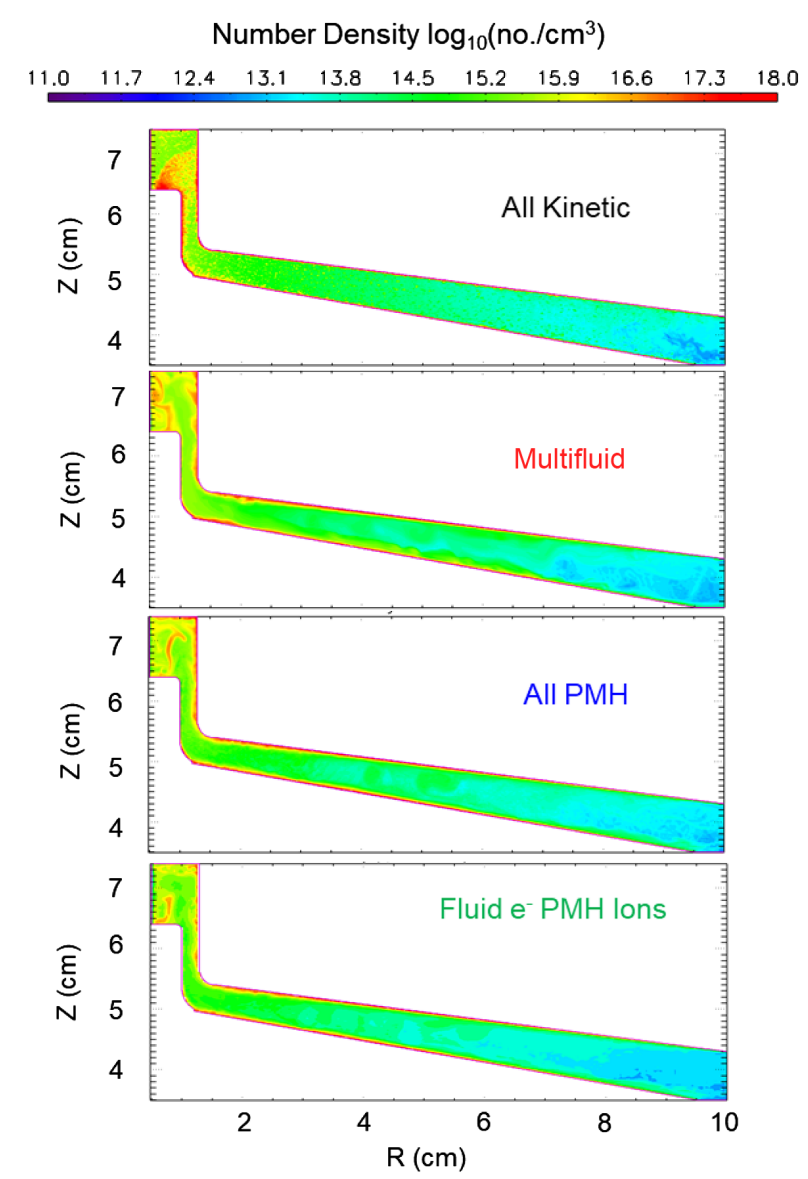

FIG. 7. The electron density is plotted 120 ns into the four 2D inner MITL simulations. The plot shows results from (a) all kinetic particles, (b) the multifluid particles, (c) all PMH with fluid particles transitioning to kinetic above $50 \mathrm{keV}$, and (d) fluid electrons with $\mathrm{PMH}$ ions. All these simulations have dynamic time steps such that $\omega_{c} \Delta t<9$.

plasma flow from the convolute at $10 \mathrm{~cm}$ radius into the simulation, we inject a linearly rising current of electrons and protons to $500 \mathrm{kA}$ at $110 \mathrm{~ns}$, constant thereafter. In the inner MITL, due to higher plasma densities evolving from the more rapidly heating electrodes, simulations must resolve the smaller skin depths as well as the larger cyclotron frequencies found near the load. We find that typically these simulations require $50 \mu \mathrm{m}$ spatial resolution for convergence but now can be run in only two dimensions.

The electron density at $120 \mathrm{~ns}$ is plotted in Fig. 7. All the simulations show roughly $10^{15} \mathrm{~cm}^{-3}$ in the radial MITL increasing to nearly $10^{16} \mathrm{~cm}^{-3}$ density in the can with a similar spray of plasma from the cathode at $z=6.4 \mathrm{~cm}$ near the load which is modeled as a short here. In the kinetic simulation, the plasma spray is more diffuse than all the fluid and hybrid simulations. Also, note the remaining dense $10^{18} \mathrm{~cm}^{-3}$ electrode plasmas along the surfaces. This indicates considerable thermal contaminant

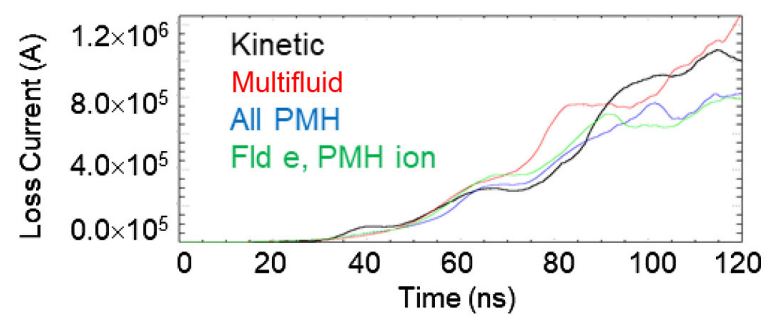

FIG. 8. The calculated current loss for four inner MITL simulations (kinetic, multifluid, $\mathrm{PMH}$, and fluid $e$ with $\mathrm{PMH}$ ions). The drive current rises to $22.6 \mathrm{MA}$ by $120 \mathrm{~ns}$.

plasma is confined there by the intense magnetic fields exceeding $1 \mathrm{MG}$ for $r<4 \mathrm{~cm}$ at least for a static load.

The $Z$ current drive at $10 \mathrm{~cm}$ radius rises to $22.6 \mathrm{MA}$ by $120 \mathrm{~ns}$. The current not transported to the load at $z=6.4 \mathrm{~cm}$ is plotted in Fig. 8. In each simulation, this loss current rises to roughly 1 MA by $120 \mathrm{~ns}$. The multifluid simulation is highest, and the two hybrid simulations' losses rise close to the kinetic curve until dropping $100 \mathrm{kA}$ below kinetic after $90 \mathrm{~ns}$. Assuming the kinetic simulation is the most accurate, it is difficult to draw many conclusions about the physics efficacy, although the PMH hybrid techniques are in line with the kinetic result for a longer period.

The relative computation speed of the multifluid simulation does have a considerable advantage. The kinetic simulation requires 38640 processor hours to run to $120 \mathrm{~ns}$ on the cluster described in Sec. V. The fluid simulation required only 11760 processor hours, a $3.3 \times$ speedup. The two hybrid simulations fell in between at 29570 (all PMH) and 14000 processor hours. These results and those of the convolute suggest the multifluid technique is a faster, reasonably accurate, alternative to all kinetic.

\section{SUMMARY AND CONCLUSIONS}

We have presented particle techniques using fluid and/or kinetic PIC macroparticle representations of plasma. The two descriptions fit nicely into the single PIC particle framework permitting migration between the two as implemented in the Lsp and Chicago codes. The more approximate fluid technique requires much fewer numbers of macroparticles than kinetic, although the solution of an additional energy equation is necessary. Also, the fluid description is better suited to plasmas at higher density and smaller temperatures that require an equation of state. As the fluid approximations (Maxwellian distributions, no particle interpenetration) become less suitable, the kinetic description using many macroparticles to represent an arbitrary velocity distribution should be used. Criteria that best determine what EOM is most suitable and least computationally expensive can be used to determine migration from fluid to kinetic and vice versa within the PMH framework. 
Using the macroparticle kinetic energy as the transition criterion for migration from fluid to kinetic, we have cross benchmarked the various techniques with previously published convolute and inner MITL models and found quite similar behavior for current loss. Subtle differences were observed relating to the turbulent nature of the electron sheath in regions of rapidly varying impedances in the outer MITLs and turbulent transport at small radii near the load. Despite these differences, all descriptions, multifluid, hybrid particles, and all kinetic particles, gave similar current losses measured just upstream of the load. The fewer particles associated with fluid and hybrid PIC representations will lead to faster turnaround in design calculations for new $Z$-pinch accelerators being considered [35-37]. Resulting designs will still need to be benchmarked against the fully kinetic treatments.

\section{ACKNOWLEDGMENTS}

The authors thank Michael Cuneo and George Laity for support of the research, Thomas Gardiner, Allen Robinson, and Matt Bettencourt for technical discussions, and Robert Clark for computational physics insights. This project was partially supported by LDRD Project No. 209240. Sandia National Laboratories is a multimission laboratory managed and operated by National Technology and Engineering Solutions of Sandia, LLC, a wholly owned subsidiary of Honeywell International, Inc., for the U.S. Department of Energy's National Nuclear Security Administration under Contract No. DE-NA0003525. This paper describes objective technical results and analysis. Any subjective views or opinions that might be expressed in the paper do not necessarily represent the views of the U.S. Department of Energy or the United States Government.

[1] C. K. Birdsall and A. B. Langdon, Plasma Physics via Computer Simulation (CRC Press, Boca Raton, FL, 2004).

[2] D. V. Rose, T. C. Genoni, R. E. Clark, D. R. Welch, and W. A. Stygar, Electron flow stability in magnetically insulated vacuum transmission lines, Phys. Plasmas 18, 033108 (2011).

[3] M. R. Gomez, R. M. Gilgenbach, M. E. Cuneo, C. A. Jennings, R. D. McBride, E. M. Waisman, B. T. Hutsel, W. A. Stygar, D. V. Rose, and Y. Maron, Experimental study of current loss and plasma formation in the $\mathrm{Z}$ machine post-hole convolute, Phys. Rev. Accel. Beams 20, 010401 (2017).

[4] B. T. Hutsel, P. A. Corcoran, M. E. Cuneo, M. R. Gomez, M. H. Hess, D. D. Hinshelwood, C. A. Jennings, G. R. Laity, D. C. Lamppa, R. D. McBride, J. K. Moore, A. Myers, D. V. Rose, S. A. Slutz, W. A. Stygar, E. M. Waisman, D. R. Welch, and B. A. Whitney, Transmission-line-circuit model of an 85-TW, 25-MA pulsed-power accelerator, Phys. Rev. Accel. Beams 21, 030401 (2018).
[5] D. R. Welch, N. Bennett, T. C. Genoni, D. V. Rose, C. Thoma, C. Miller, and W. A. Stygar, Electrode contaminant plasma effects in $10^{7}-A \mathrm{Z}$ pinch accelerators, Phys. Rev. Accel. Beams 22, 070401 (2019).

[6] D. R. Welch, D. V. Rose, N. Bruner, R. E. Clark, B. V. Oliver, K. D. Hahn, and M. D. Johnston, Hybrid simulation of electrode plasmas in high-power diodes, Phys. Plasmas 16, 123102 (2009).

[7] J. U. Brackbill and H. M. Ruppel, FLIP: A method for adaptively zoned, particle-in-cell calculations of fluid flows in two dimensions, J. Comput. Phys. 65, 314 (1986).

[8] S. Markidis, V. Olshevsky, C. P. Sishtla, S. W. D. Chien, E. Laure, and G. Lapenta, PolyPIC: The polymorphic-particle-in-cell method for Fluid-Kinetic coupling, Front. Phys. 04, 100 (2018).

[9] W. A. Stygar, M. E. Cuneo, D. I. Headley, H. C. Ives, R. J. Leeper, M. G. Mazarakis, C. L. Olson, J. L. Porter, T. C. Wagoner, and J. R. Woodworth, Architecture of petawattclass z-pinch accelerators, Phys. Rev. Accel. Beams 10, 030401 (2007).

[10] D. H. McDaniel, M. G. Mazarakis, D. E. Bliss, J. M. Elizondo, H. C. Harjes, H. C. Ives, D. L. Kitterman, J. E. Maenchen, T. D. Pointon, S. E. Rosenthal, D. L. Smith, K. W. Struve, W. A. Stygar, E. A. Weinbrecht, D. L. Johnson, and J.P. Corely, in Proceedings of the 5th International Conference on Dense Z-Pinches, edited by J. Davis (AIP, New York, 2002), p. 23.

[11] M. E. Savage et al., in Proceedings of the 16th IEEE Pulsed Power and Plasma Science Conference Albuquerque, NM (IEEE, New York, 2007), p. 979.

[12] D. V. Rose, D. R. Welch, E. A. Madrid, C. L. Miller, R. E. Clark, W. A. Stygar, M. E. Savage, G. A. Rochau, J. E. Bailey, T. J. Nash, M. E. Sceiford, and K. W. Struve, Threedimensional electromagnetic model of the pulsed-power Zpinch accelerator, Phys. Rev. Accel. Beams 13, 010402 (2010).

[13] K. Yee, Numerical solution of initial boundary value problems involving Maxwell's equations in isotropic media, IEEE Trans. Antennas Propag. 14, 302 (1966).

[14] D. R. Welch, D. V. Rose, B. V. Oliver, and R. E. Clark, Simulation techniques for heavy ion fusion chamber transport, Nucl. Instrum. Methods Phys. Res., Sect. A 464, 134 (2001).

[15] T. Zh. Esirkepov, Exact charge conservation scheme for Particle-in-Cell simulation with an arbitrary form-factor, Comput. Phys. Commun. 135, 144 (2001).

[16] T. D. Pointon, W. L. Langston, and M. E. Savage, Computer simulations of the magnetically insulated transmission lines and post-hole convolute of ZR, Report No. SAND2007-6466C, 2007.

[17] B. A. Trubnikov, Particle interactions in a fully-ionized plasma, in Reviews of Plasma Physics (Plenum, New York, 1965), Vol. 1, p. 105.

[18] K. Nanbu and S. Yonemura, Weighted particles in coulomb collision simulations based on the theory of a cumulative scattering angle, J. Comput. Phys. 145, 639 (1998); K. Nanbu, Theory of cumulative small-angle collisions in plasmas, Phys. Rev. E 55, 4642 (1997).

[19] D. P. Higginson, I. Holod, and A. Link, A corrected method for Coulomb scattering in arbitrarily weighted 
particle-in-cell plasma simulations, J. Comput. Phys. 413, 109450 (2020).

[20] C. Thoma, D. R. Welch, R. E. Clark, N. Bruner, J. J. MacFarlane, and I. E. Golovkin, Two-fluid electromagnetic simulations of plasma-jet acceleration with detailed equation-of-state, Phys. Plasmas 18, 103507 (2011).

[21] D. R. Welch, D. V. Rose, M. E. Cuneo, R. B. Campbell, and T. A. Mehlhorn, Integrated simulation of the generation and transport of proton beams from laser-target interaction, Phys. Plasmas 13, 063105 (2006).

[22] C. Thoma, D. R. Welch, and S. C. Hsu, Particle-in-cell simulations of collisionless shock formation via head-on merging of two laboratory supersonic plasma jets, Phys. Plasmas 20, 082128 (2013).

[23] D. R. Welch, D. V. Rose, R. E. Clark, C. B. Mostrom, W. A. Stygar, and R. J. Leeper, Fully Kinetic Particle-inCell Simulations of a Deuterium Gas Puff Z-Pinch, Phys. Rev. Lett. 103, 255002 (2009).

[24] C. Thoma, T. C. Genoni, D. R. Welch, D. V. Rose, R. E. Clark, C. L. Miller, W. A. Stygar, and M. L. Kiefer, Numerical simulation of cathode plasma dynamics in magnetically insulated vacuum transmission lines, Phys. Plasmas 22, 032101 (2015).

[25] D. W. Hewitt and A. B. Langdon, Electromagnetic direct implicit plasma simulation, J. Comput. Phys. 72, 121 (1987).

[26] T. C. Genoni, R. E. Clark, and D. R. Welch, A fast implicit algorithm for highly magnetized charged particle motion, Open Plasma Phys. J. 3, 36 (2010).

[27] C. Thoma, D. R. Welch, R. E. Clark, D. V. Rose, and I. E. Golovkin, Hybrid-PIC modeling of laser-plasma interactions and hot electron generation in gold hohlraum walls, Phys. Plasmas 24, 062707 (2017).

[28] H. Knoepfel, Magnetic Fields (John Wiley and Sons, New York, 2000).
[29] M. Li and H. F. Dylla, Model for the outgassing of water from metal surfaces, J. Vac. Sci. Technol. A 11, 1702 (1993).

[30] H. F. Dylla, D. M. Manos, and P. H. LaMarche, Correlation of outgassing of stainless steel and aluminum with various surface treatments, J. Vac. Sci. Technol. A 11, 2623 (1993).

[31] A Comptant La Fontaine, Ion emission at the target of the radiographic devices PIVAIR and AIRIX, J. Phys. D 40, 1712 (2007).

[32] D. V. Rose, E. A. Madrid, D. R. Welch, R. E. Clark, C. B. Mostrom, W. A. Stygar, and M. E. Cuneo, Computational analysis of current-loss mechanisms in a post-hole convolute driven by magnetically insulated transmission lines, Phys. Rev. Accel. Beams 18, 030402 (2015).

[33] B. W. Church and R. N. Sudan, A self-consistent quasistatic equilibrium for non-neutral diamagnetic electron vortices, Phys. Plasmas 2, 1837 (1995).

[34] N. Bennett, D. R. Welch, C. A. Jennings, E. Yu, M. H. Hess, B. T. Hutsel, G. Laity, J. K. Moore, D. V. Rose, K. Peterson, and M.E. Cuneo, Current transport and loss mechanisms in the $\mathrm{Z}$ accelerator, Phys. Rev. Accel. Beams 22, 120401 (2019).

[35] W. A. Stygar, M. E. Cuneo, D. I. Headley, H. C. Ives, R. J. Leeper, M. G. Mazarakis, C. L. Olson, J. L. Porter, and T. C. Wagoner, Architecture of petawatt-class z-pinch accelerators, Phys. Rev. Accel. Beams 10, 030401 (2007).

[36] D. R. Welch, T. C. Genoni, D. V. Rose, N. Bruner, and W. A. Stygar, Optimized transmission-line impedance transformers for petawatt-class pulsed-power accelerators, Phys. Rev. Accel. Beams 11, 030401 (2008).

[37] W. A. Stygar et al., Conceptual designs of two petawattclass pulsed-power accelerators for high-energy-densityphysics experiments, Phys. Rev. Accel. Beams 18, 110401 (2015). 